\title{
Embedded RF Test Circuits: RF Power Detectors, RF Power Control Circuits, Directional Couplers, and 77-GHz Six-Port Reflectometer
}

\author{
William R. Eisenstadt ${ }^{*}$ and Byul Hur, Member, KIICE \\ Department of Electrical and Computer Engineering, University of Florida, Gainesville, FL 32611, USA
}

\begin{abstract}
Modern integrated circuits (ICs) are becoming an integrated parts of analog, digital, and radio frequency (RF) circuits. Testing these RF circuits on a chip is an important task, not only for fabrication quality control but also for tuning RF circuit elements to fit multi-standard wireless systems. In this paper, RF test circuits suitable for embedded testing are introduced: RF power detectors, power control circuits, directional couplers, and six-port reflectometers. Various types of embedded RF power detectors are reviewed. The conventional approach and our approach for the RF power control circuits are compared. Also, embedded tunable active directional couplers are presented. Then, six-port reflectometers for embedded RF testing are introduced including a 77-GHz six-port reflectometer circuit in a $130 \mathrm{~nm}$ process. This circuit demonstrates successful calibrated reflection coefficient simulation results for 37 well distributed samples in a Smith chart. The details including the theory, calibration, circuit design techniques, and simulations of the 77-GHz six-port reflectometer are presented in this paper.
\end{abstract}

Index Terms: Built-in self-test, Directional couplers, Embedded RF test, Microwave measurements, Reflectometry

\section{INTRODUCTION}

Modern personal communication electronics demand the integration of analog, digital, and radio frequency (RF) circuits on a chip. It is common to find these integrated circuits (ICs) inside cell phones, laptops, tablets, and personal computers (PCs). It is important to embed RF circuits not only for improved fabrication quality control but also for tuning of the RF circuit elements to fit multistandard wireless communication platforms. The essential embedded RF test circuits introduced in this paper are the RF power detectors, RF power control circuits, directional couplers, and six-port reflectometers (SPRs). The embedded $\mathrm{RF}$ test circuits are generally simple and compact. The conventional approaches and our new approach for these important embedded RF test and RF tuning circuits are introduced in this paper.

\section{RF POWER DETECTORS}

RF power detectors are the primary test elements in embedded RF testing systems. The RF power detectors convert input RF power signals to representative DC voltages. The power detectors can be implemented as peak detectors (envelop detectors) or root-mean-square (RMS) detectors $[1,2]$. The peak detector operations can be understood simply as a combination of a diode and a

\footnotetext{
Received 25 September 2012, Revised 10 December 2012, Accepted 26 December 2012

*Corresponding Author William R. Eisenstadt (E-mail: wre@tec.ufl.edu, Tel: +1-352-392-4946)

Department of Electrical and Computer Engineering, University of Florida, 925 NW 56th Terrace, Gainesville, FL 32611, USA.
}

(c) This is an Open Access article distributed under the terms of the Creative Commons Attribution Non-Commercial License (http://creativecommons.org/li-censes/by$\mathrm{nc} / 3.0 /$ ) which permits unrestricted non-commercial use, distribution, and reproduction in any medium, provided the original work is properly cited. 
capacitor. The input RF signals are tailored by the diode. Then, the capacitor stores the peak value of the charge. Depending on the circuit design techniques, the DC levels can be measured as peak or RMS values. RMS RF power detectors may be found to be more useful than peak detectors in many wireless applications. However, generally, RMS detectors are more complex and require a larger chip area. RMS power detectors can be implemented using thermal detection, diode detection, or translinear detection methods [3]. Thermal-based RF power detectors convert the input RF power to the thermal power and measure the ambient temperature changes [4]. The detectors can achieve wide bandwidth and good accuracy. However, the detectors may not be a good candidate for embedded testing because of the thermal coupling from the adjacent circuits. Diode detectors can be implemented as RF RMS detectors by exploiting their square-law characteristic in a small signal operation region [5]. However, the circuits require Schottky diodes, which are not available in a standard CMOS process. Translinear RF detectors have V-I conversion and squarerdivider circuits. Previous research demonstrated that reasonably sized translinear RF detectors are suitable for embedded RF testing [6]. However, translinear circuits can have a limited frequency response below $1 \mathrm{GHz}$.

\section{RF POWER CONTROL CIRCUITS}

Input and output RF power may be attenuated or amplified in many wireless applications. One power control method is to build automatic gain control (AGC) systems. These AGC systems can achieve RF power control with fine gain steps and good dynamic ranges [7,8]. However, they tend to require a large chip area. Large area circuits are poor candidates for embedded RF testing.

Variable gain amplifiers (VGAs) are parts of the AGC and, they can be used as a single element to control RF power as an open-loop system [9]. Where the type of gain control input for a VGA is digital, the circuit is called a digital variable gain amplifier (DVGA) [10]. In certain wireless applications, it is important to make the gain control circuits broadband. One of the methods is to utilize distributed amplifiers [11].

We have developed a mixed system with distributed amplifiers and digital gain control circuits resulting in broadband, digital, and fine gain control steps. A block diagram of the programmable gain distributed amplifier (PGDA) is shown in Fig. 1. Two RF lines are utilized, and the circuit has three cells, where each cell has one or more digitally controllable amplifiers. The middle cell has multiple amplifiers, and the cell can generate various digitally controlled transconductances. One of the published PGDAs is the CMOS PGDA with $0.5 \mathrm{~dB}$ gain steps [12].

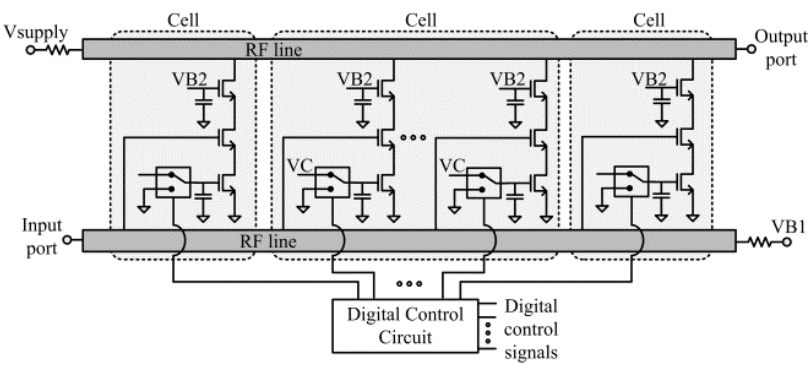

Fig. 1. Simplified block diagram of a programmable gain distributed amplifier.

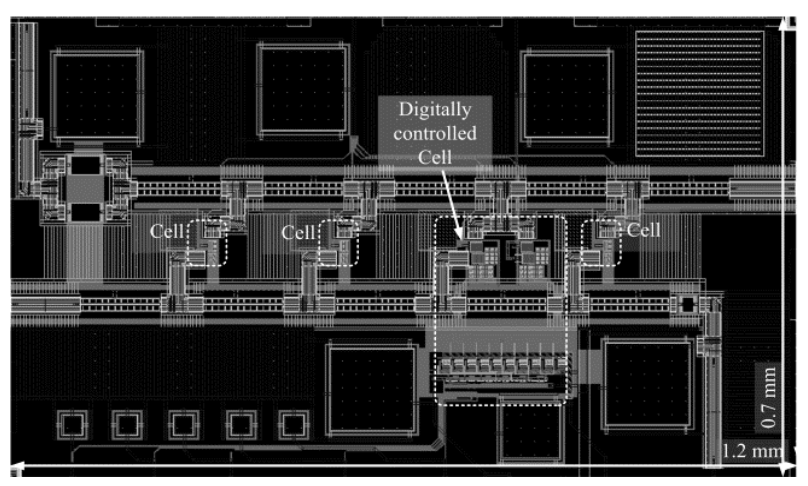

Fig. 2. Layout (core) of the programmable gain distributed amplifier with controlled $0.5 \mathrm{~dB}$ gain steps in a $130 \mathrm{~nm}$ BiCMOS process.

It is designed, fabricated, and measured successfully using a $130 \mathrm{~nm}$ process consisting of four cells, where each cell includes an amplifier block.

The amplifier block in the 3rd cell contains multiple amplifiers, where the amplifiers are controlled digitally by the digital control circuit block. The input signals for the digital control block are 3 bit digital signals and a clock signal. The control signals generated by the digital control block make each amplifier active or inactive. Then, the total transconductance gets varied digitally because it is equal to the summation of all the transconductance of the cells. The core layout of the chip using a Cadence IC design tool is shown by a screenshot in Fig. 2. The core chip area is $1.2 \mathrm{~mm} \times 0.7 \mathrm{~mm}$. The area of the chip can be reduced, by $33 \%$ of the vertical and $80 \%$ of the horizontal length of Fig. 2.

\section{DIRECTIONAL COUPLERS}

The directional couplers are the core elements for reflection of the measurement circuits. They are generally fabricated as coupled microwave lines. However, in low frequency applications, these coupled microwave lines take too large a chip area for embedded test circuits. 


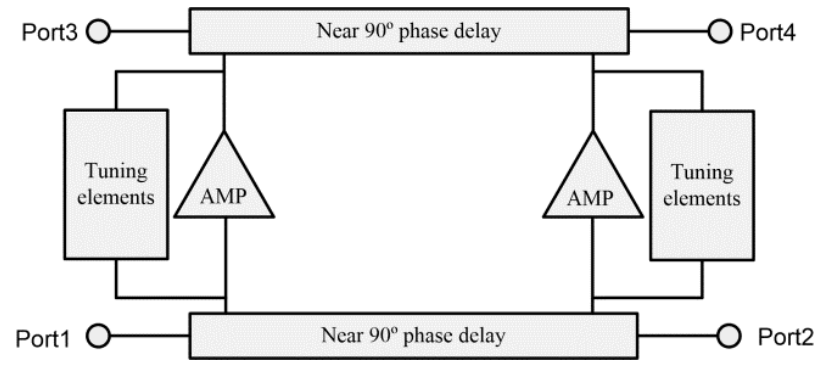

Fig. 3. Simplified generalized block diagram of a tunable active directional coupler. AMP: amplifier.

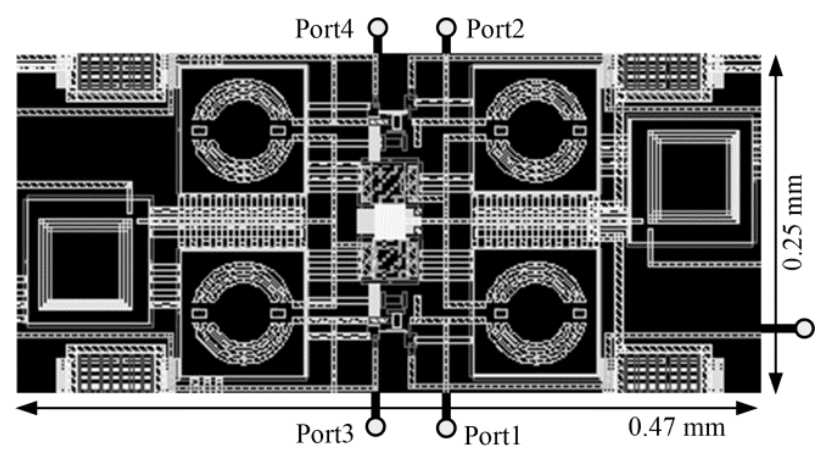

Fig. 4. Layout (Core) of the tunable active directional coupler.

Therefore, lumped directional couplers may be a better choice because they take less chip area for low frequency applications [13, 14]. Moreover, by making the directional couplers tunable, they become more flexible and fit many wireless standards [15]. It is a challenge to design tunable directional couplers that are both broadband and highly reconfigurable.

The authors have developed tunable broadband active directional couplers that are suitable for embedded RF testing to satisfy both needs of being broadband and highly reconfigurable [16]. A simplified generalized block diagram of the tunable active directional coupler is described in Fig. 3 . There are two amplifiers and tuning elements connected between two near $90^{\circ}$ phase delays. The near $90^{\circ}$ phase delay represents the RF elements and introduces a phase shift of approximately $90^{\circ}$. Circuit parasitics and variable capacitors from the tuning elements will change the degree of the phase delay between the RF lines while the coupler circuit is tuned. If one applies the input signals at port 1 , port 2 is a through port with $90^{\circ}$ phase shifted signals from the input signals. Port 4 is a constructive port combining the signals from port 1 and port 2 . However, port 3 is a deconstructive port cancelling out the signals from port 2 . The detailed theoretical background of this type of directional coupler can be found in [16], where it shows the specific design, fabrication, and measurement of a tunable active directional coupler circuit with $2.4 \mathrm{GHz}$ bandwidth broadband. The core layout of the chip, a screenshot using the Cadence IC design tool, is shown in Fig. 4, where the area is $0.47 \mathrm{~mm} \times 0.25 \mathrm{~mm}$. The phase shifters are implemented as two inductors and capacitors. One of the phase shifters is located in between port 1 and port 2, and the other phase shifter is between port 3 and port 4 . There are also two more small size on-chip bias inductors found in Fig. 4.

\section{SIX-PORT REFLECTOMETERS}

\section{A. Reflectometer for Embedded RF Testing}

Vector network analyzers (VNAs) are widely used to measure reflection coefficients and gain. The VNAs include RF power detectors, a power control system, directional couplers, and other types of RF circuits. However, conventional VNAs have fairly complicated hardware and software, and they may consume a large amount of chip space, which makes them unsuitable for the embedded RF test. Reflectometers are devices that can measure signal reflections. One of the candidates for reflectometers suitable for embedded RF testing is a SPR. The primary advantages is that SPRs have is a simple circuit structure. However, the calibration method of SPRs is more complicated than conventional VNA error correction.

\section{B. Theory and Calibration of the SPR}

The SPR was popularized by Engen $[17,18]$ and others. One published SPR on-a-chip can work from 1.3 to $3.0 \mathrm{GHz}$ [19]. The authors have developed on-chip SPR systems at high frequencies. The block diagram of a $77-\mathrm{GHz}$ SPR is shown in Fig. 5. It has one divider, one phase shifter, three single ended RF power detectors (D3, D5, and D6), and one differential RF power detector (D4). The details of the single-ended and differential RF detectors can be found in Widemann and colleagues' paper [19]. And, their SPR calibration method was adopted [20]. The SPR calibration takes the following steps. First, the detectors are characterized properly by changing the input power levels. This method of in situ detector characterization is essential, where the in situ detector characterization means that the detectors are characterized without disconnecting them from the circuit [21]. Next, initial values are estimated for the calibration constants. There are various methods of finding these initial values in the literature [22-24]. The method in this research use a minimum of five unknown loads with constant magnitude reflection coefficients and well-distributed phases [20]. 


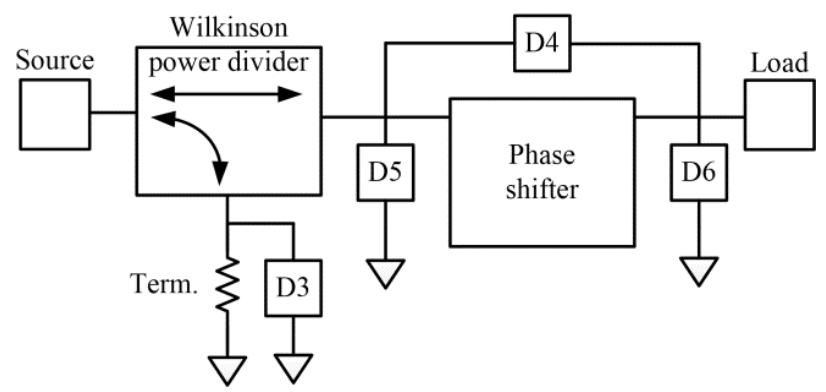

Fig. 5. Block diagram of a 77-GHz six-port reflectometer.

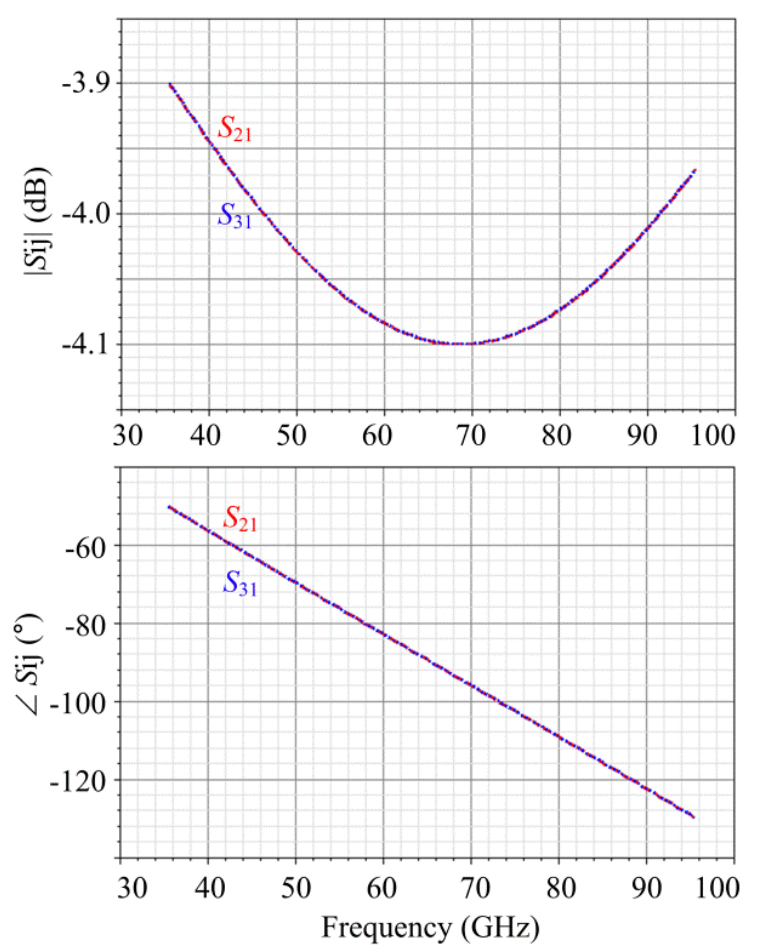

Fig. 6. Simulations of the Power divider: magnitude (upper graph) and phase (lower graph) plots of $S_{21}$ and $S_{31}$.

The next step is to perform a six- to four-reductions to decrease the number of the required coefficients of the SPR. A SPR requires eleven coefficients; however, a four-port reflectometer requires six coefficients. Engen presents an approach to perform calibration in a reduced four-port reflectometer [22].

The next step is to carry out an "error box" transformation, which is a mapping technique for the reflection coefficient from the $\mathrm{W}$ plane to the $\Gamma$ plane. The important variables of an SPR calibration, $a, b$, and c, can be found using three known loads and by solving three linear equations. There are also other calibration methods reported in the literature $[25,26]$.

\section{77-GHz SPR Design, Simulation, and Verification}

The spectrum range of the W-band is from 75 to $110 \mathrm{GHz}$. A frequency of around $77 \mathrm{GHz}$ is generally utilized for automotive radar system applications. This section presents an on-chip SPR targeted at $77 \mathrm{GHz}$. The circuit is designed using a $130 \mathrm{~nm}$ process, as shown in Fig. 5. For the power divider, a microstrip Wilkinson power divider was chosen and designed to work at $77 \mathrm{GHz}$.

The simulation results, where the power injected to port 1 is divided into port 2 and port 3, are shown in Fig. 6. For the phase shifter, a RF transmission line is utilized since the frequency is high enough that the RF line is short.

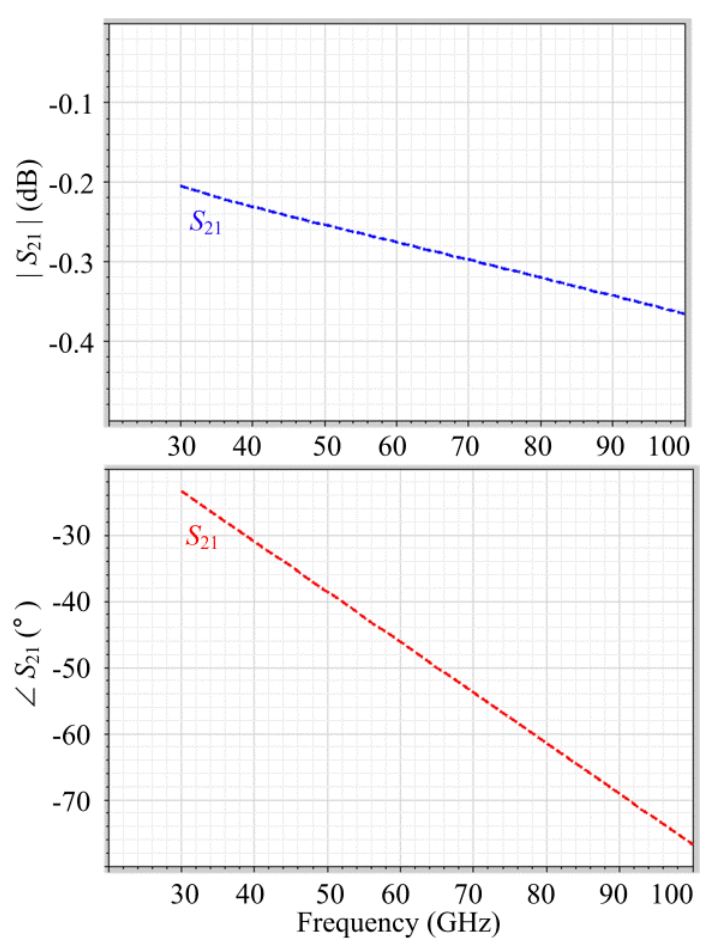

Fig. 7. Simulations of the phase shifter: magnitude (upper graph) and phase (lower graph) plots of $S_{21}$.

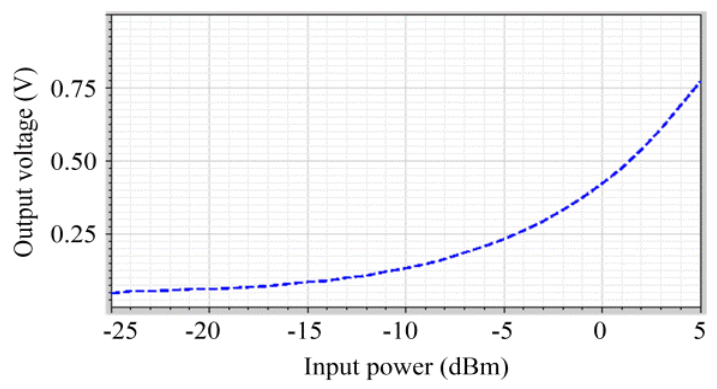

Fig. 8. Simulation results of the sweeping input power of a single-ended detector from -25 to $5 \mathrm{dBm}$ at $77 \mathrm{GHz}$. 


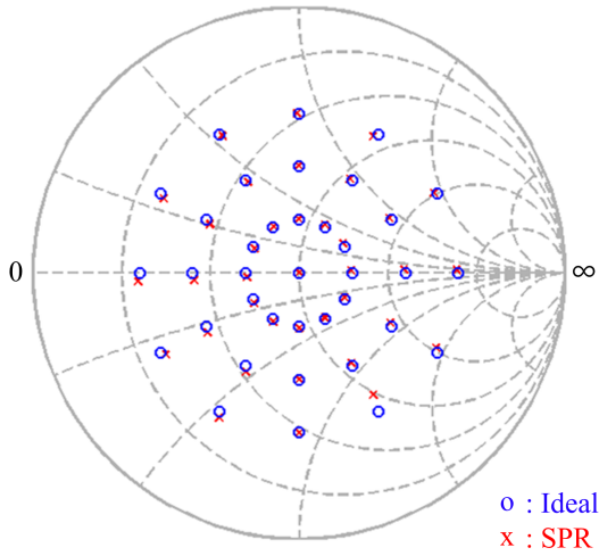

Fig. 9. Simulation results of $77-\mathrm{GHz}$ six-port reflectometer (SPR) at 77 $\mathrm{GHz}$ with selected test points. "o" represents ideal reflection coefficients, and " $x$ " represents the calibrated reflection coefficients using the SPR .

The simulations of the phase shifter are shown in Fig. 7. The phase at $77 \mathrm{GHz}$ is approximately $60^{\circ}$. For the RF power detectors, Lee and Eisenstadt's power detectors are chosen [27]. Their paper demonstrated detectors working up to $80 \mathrm{GHz}$ in simulation and measuring up to $67 \mathrm{GHz}$. The simulation results for the single-ended RF power detector demonstrate a power sweep from -25 to $5 \mathrm{dBm}$ at $77 \mathrm{GHz}$, as shown in Fig. 8. The simulated reflection coefficient measurement results for 37 well-distributed sample points are shown in Fig. 9. Good agreements are shown in the reflection coefficients between the ideal tuner and the SPR results.

However, a few of the data points do not show good agreement because minor errors in the periodic steady-state (PSS) analysis simulations cause the errors since PSS has a tendency to simplify the simulation process and to amplify minor numerical errors to increase the speed of the simulation.

\section{CONCLUSIONS}

The embedded RF test circuits, RF power detectors, power control circuits, directional couplers, and a $77-\mathrm{GHz}$ SPR, have been introduced. RF power detectors for embedded RF testing were presented. RF power control circuits were introduced, and directional couplers were presented. The design, theory and calibration techniques for a 77-GHz SPR were detailed. Finally, successful results were demonstrated in simulations. As the importance of testing RF circuits on a chip is increasing, the test circuits for embedded RF testing including the circuits introduced in this paper have wide applicability for modern IC designs.

\section{ACKNOWLEDGMENTS}

The PGDA research in Section III was supported by the Focus Center Research Program (FCRP) Center for Circuit and System Solutions (C2S2) (Theme: 888.019, August 2008-July 2009). The tunable active directional coupler research in Section IV was supported by SRC Global Research Collaboration (GRC) program and Freescale Semiconductor (Theme: 1663.001 and 1836.026, January 2009-June 2010). The preliminary six-port reflectometer research including theory and calibration was supported by National Science Foundation (NSF) (SBIR/2010, Contract No. 1013695).

\section{REFERENCES}

[1] R. G. Meyer, "Low-power monolithic RF peak detector analysis," IEEE Journal of Solid-State Circuits, vol. 30, no. 1, pp. 65-67, 1995.

[2] A. Valdes-Garcia, R. Venkatasubramanian, R. Srinivasan, J. SilvaMartinez, and E. Sanchez-Sinencio, "A CMOS RF RMS detector for built-in testing of wireless transceivers," in Proceedings of the 23rd IEEE VLSI Test Symposium, Palm Springs: CA, pp. 249- 254, 2005.

[3] Y. Zhou and M. Y. W. Chia, "A low-power ultra-wideband CMOS true RMS power detector," IEEE Transactions on Microwave Theory and Techniques, vol. 56, no. 5, pp. 1052-1058, 2008.

[4] E. H. Klaassen, R. J. Reay, and G. T. A. Kovacs, "Diode-based thermal RMS converter with on-chip circuitry fabricated using standard CMOS technology," in Proceedings of the 8th International Conference on Solid-State Sensors and Actuators (TRANSDUCERS), Stockholm, Sweden, pp.154-157, 1995.

[5] L. Lei, J. L. Hesler, H. Xu, A. W. Lichtenberger, and R. M. Weikle, "A broadband quasi-optical terahertz detector utilizing a zero bias Schottky diode," IEEE Microwave and Wireless Components Letters, vol. 20, no. 9, pp. 504-506, 2010.

[6] Q. Yin, W. R. Eisenstadt, R. M. Fox, and T. Zhang, "A translinear RMS detector for embedded test of RF ICs," IEEE Transactions on Instrumentation and Measurement, vol. 54, no. 5, pp. 1708-1714, 2005.

[7] D. V. Mercy, "A review of automatic gain control theory," Radio and Electronic Engineer, vol. 51, no. 11/12, pp. 579-590, 1981.

[8] Q. H. Duong, Q. Le, C. W. Kim, and S. G. Lee, “A 95-dB linear low-power variable gain amplifier," IEEE Transactions on Circuits and Systems I: Regular Papers, vol. 53, no. 8, pp. 1648-1657, 2006.

[9] H. D. Lee, K. A. Lee, and S. C. Hong, "A wideband CMOS variable gain amplifier with an exponential gain control," IEEE Transactions on Microwave Theory and Techniques, vol. 55, no. 6, pp. 1363-1373, 2007.

[10] H. O. Elwan, A. El Adawi, M. Ismail, H. K. Olsson, and A. M. Soliman, "Digitally controlled dB-linear CMOS variable gain amplifier," Electronics Letters, vol. 35, no. 20, pp. 1725-1727, 1999. 
[11] F. Zhang and P. R. Kinget, "Low-power programmable gain CMOS distributed LNA," IEEE Journal of Solid-State Circuits, vol. 41, no. 6, pp. 1333-1343, 2006.

[12] B. Hur and W. R. Eisenstadt, "CMOS programmable gain distributed amplifier with 0.5 -dB gain steps," IEEE Transactions on Microwave Theory and Techniques, vol. 59, no. 6, pp. 15521559, 2011.

[13] R. W. Vogel, "Analysis and design of lumped- and lumpeddistributed-element directional couplers for MIC and MMIC applications," IEEE Transactions on Microwave Theory and Techniques, vol. 40, no. 2, pp. 253-262, 1992.

[14] Y. C. Chiang and C. Y. Chen, "Design of a wide-band lumpedelement 3-dB quadrature coupler," IEEE Transactions on Microwave Theory and Techniques, vol. 49, no. 3, pp. 476-479, 2001.

[15] E. A. Fardin, K. Ghorbani, and A. S. Holland, "A varactor tuned branch-line hybrid coupler," in Proceedings of Asia-Pacific Microwave Conference (APMC2005), Suzhou, China, 2005.

[16] B. Hur and W. R. Eisenstadt, "Tunable broadband MMIC active directional coupler," IEEE Transactions on Microwave Theory and Techniques, vol. 61, no. 1, pp. 168-176, 2013.

[17] G. F. Engen, "The six-port reflectometer: an alternative network analyzer," in IEEE MTT-S International Microwave Symposium Digest, San Francisco: CA, pp.44-46, 1977.

[18] G. F. Engen, "A (historical) review of the six-port measurement technique," IEEE Transactions on Microwave Theory and Techniques, vol. 45, no. 12, pp. 2414-2417, 1997.

[19] F. Wiedmann, B. Huyart, E. Bergeault, and L. P. Jallet, "New structure for a six-port reflectometer in monolithic microwave integrated-circuit technology," IEEE Transactions on Instrumentation and Measurement, vol. 46, no. 2, pp. 527-530, 1997.
[20] F. Wiedmann, B. Huyart, E. Bergeault, and L. P. Jallet, "A new robust method for six-port reflectometer calibration," IEEE Transactions on Instrumentation and Measurement, vol. 48, no. 5, pp. 927-931, 1999.

[21] E. Bergeault, B. Huyart, G. P. J. Geneves, and L. P. Jallet, "Characterization of diode detectors used in six-port reflectometers," IEEE Transactions on Instrumentation and Measurement, vol. 40, no. 6, pp. 1041-1043, 1991.

[22] G. F. Engen, "Calibrating the six-port reflectometer by means of sliding terminations," IEEE Transactions on Microwave Theory and Techniques, vol. 26, no. 12, pp. 951-957, 1978.

[23] U. Stumper, "Finding initial estimates needed for the Engen method of calibrating single six-port reflectometers," IEEE Transactions on Microwave Theory and Techniques, vol. 38, no. 7, pp. 946-949, 1990.

[24] B. Neumeyer, "A new analytical method for complete six-port reflectometer calibration," IEEE Transactions on Instrumentation and Measurement, vol. 39, no. 2, pp. 376-379, 1990.

[25] I. Kasa, "Closed-form mathematical solutions to some network analyzer calibration equations," IEEE Transactions on Instrumentation and Measurement, vol. 23, no. 4, pp. 399-402, 1974.

[26] H. J. Eul and B. Schiek, "A generalized theory and new calibration procedures for network analyzer self-calibration," IEEE Transactions on Microwave Theory and Techniques, vol. 39, no. 4, pp. 724-731, 1991.

[27] M. C. Lee and W. R. Eisenstadt, "A wide-band differential and single-ended microwave amplitude detector," in Proceedings of the 11th Annual IEEE Wireless and Microwave Technology Conference, Melbourne: FL, pp.1-5, 2010.

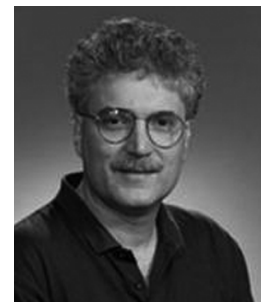

William R. Eisenstadt

received the B.S., M.S., and Ph.D. degrees in electrical engineering from Stanford University, Stanford, CA, in 1979, 1981, and 1986, respectively. In 1984, he joined the faculty of the University of Florida, Gainesville, where he is currently a Professor. He has authored or coauthored over 150 refereed conference and journal publications. His research focuses on mixed-signal/RF embedded integrated circuit (IC) testing, high-speed I/O characterization, BiSTs, and differential S-parameter characterization of integrated circuit (IC) devices, packages, and interconnection. In addition, he has been involved with large-signal microwave circuit design and test and power-amplifier design. $\mathrm{He}$ has over 30 years of experience in IC design and testing. Dr. Eisenstadt serves on the ISCAS Analog Signal Processing Technical Committee and the Wireless Test Workshop Executive Committee. He was the recipient of the 1985 National Science Foundation Presidential Young Investigator Award.

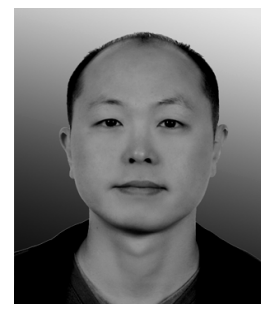

\section{Byul Hur}

received the B.S. degree in electronics engineering from Yonsei University, Seoul, Korea, in 2000, and the M.S. and Ph.D. degrees from the University of Florida, Gainesville, Florida in 2007 and 2011, respectively. $\mathrm{He}$ is currently a postdoctoral associate researcher at the University of Florida. His research has focused on mixed-signal/RF embedded integrated circuit (IC) design and testing, and he is an experienced research engineer who holds versatile skills in both hardware and software from research to product. 